\title{
Simulation of TRT-Configured Ground-Penetrating Radars Over Heterogeneous Grounds
}

\author{
UĞUR OĞUZ* AND LEVENT Güret \\ DEPARTMENT OF ELECTRICAL AND ELECTRONICS ENGINEERING \\ BILKENT UNIVERSITY \\ BILKENT, ANKARA, TURKEY \\ (uoguz@cem.bilkent.edu.tr, lgurel@ee.bilkent.edu.tr)
}

\section{Introduction}

The finite-difference time-domain (FDTD) method, along with the perfectlymatched layer (PML) absorbing boundary conditions (ABCs), has been used extensively to simulate ground-penetrating-radar (GPR) scenarios. The main difficulty in both practical and numerical GPR problems is the domination of the received signals by the direct coupling from the transmitter. Various practical [1] and numerical [2] methods have been devised to degrade this coupling and enhance the detectability of the buried target.

The transmitter-receiver-transmitter (TRT) configuration $[3,4]$ is an attempt to design GPR models with identically zero coupling at the receiver. In this configuration, the receiving antenna is located in the middle of the two identical transmitters, which are fed $180^{\circ}$ out of phase. The TRT configuration, illustrated in Fig. 1(a), implies the existence of a symmetry plane in the middle two transmitters and the cancellation of the direct signals $\left(D_{1}\right.$ and $\left.D_{2}\right)$ coupled from the transmitters at the receiver location. Moreover, if the ground is homogeneous and the ground-air interface is uniform, the two reflected signals $\left(G_{1}\right.$ and $\left.G_{2}\right)$ also cancel out at the receiver. The TRT configuration is an efficient and powerful way to enhance the detection of buried targets by removing or decreasing the amplitudes of large undesired signals.

Although different alignment and polarizations of TRT-configured GPR models yield a variety of results on a fixed scenario [4], in this paper, a single configuration, which is depicted in Fig. 1(b), is used. This GPR model consists of three horizontally-polarized antennas aligned parallel to the path of the radar unit.

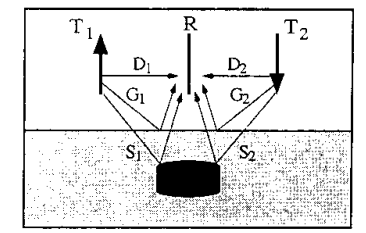

(a)

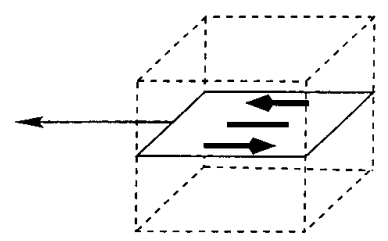

(b)

Figure 1: (a) Basic TRT configuration of the radar unit and the definition of the direct $\left(D_{1}\right.$, and $\left.D_{2}\right)$, reflected $\left(G_{1}\right.$, and $\left.G_{2}\right)$, and scattered $\left(S_{1}\right.$, and $\left.S_{2}\right)$ signals. (b) The TRT configuration used in this paper. 


\section{Optimization of the Antenna Separation}

For the GPR configuration in Fig. 1(b), changing the distance between the transmitting and receiving antennas affects the amount of scattered energy observed at the receiver. Figure 2 displays two C-scan results of a conducting disk, uitl $2.5 \mathrm{~cm}$ radius, $4 \mathrm{~cm}$ height, and buried $5 \mathrm{~cm}$ under the ground. These two results are obtained with GPR models of $1-\mathrm{cm}$ and $4-\mathrm{cm}$ transmitter-receiver (T-R) separations. Figures 2(a) and (b) reveal that larger amounts of scattered energy is observed on the receiver while the $T-R$ separation increases.

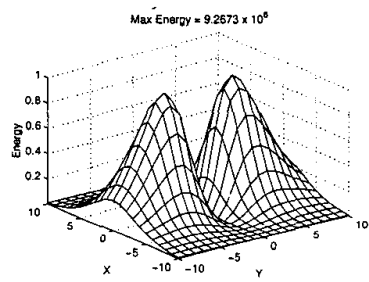

(a)

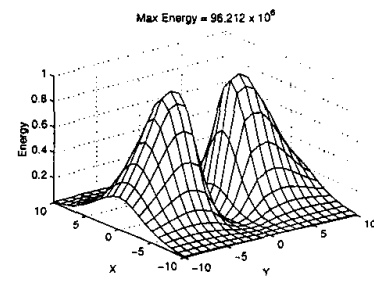

(b)

Figure 2: The C-scan results of a conducting disk, buried $5 \mathrm{~cm}$ under the ground. The T-R separation is (a) $1 \mathrm{~cm}$ and (b) $4 \mathrm{~cm}$.

Although the received energy seemed to increase with the separation of the antennas in Fig. 2, the received scattered energy is likely to decrease to zero as the separation approaches infinity. Therefore, an optimum value should be encountered while the transmitters and the receiver are taken apart. In order to demonstrate the existence of this optimum distance and find its value, a number of simulations are carried out with the TRT-configured GPR model. In the referred simulations, the T-R separation of the GPR model is changed from $1 \mathrm{~cm}$ to $16 \mathrm{~cm}$ in one-cm steps. With each of the $16 \mathrm{GPR}$ models, a B-scan measurement is performed and the maximum received energy is recorded. Figure 3 displays these energy figures with respect to the T-R separation value and demonstrates that the optirnum T-R separation value is between $6 \mathrm{~cm}$ and $7 \mathrm{~cm}$

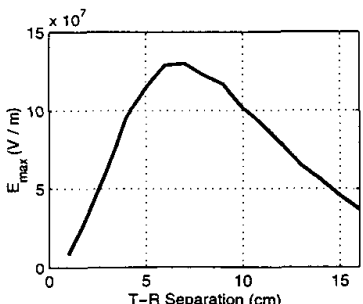

Figure 3: The maximum received energy vs. the $T-R$ separation. 


\section{B-Scan Results with Heterogeneous Ground Model}

In this section, the performance of the TRT-configured GPR model is investigated when operating on heterogeneons ground models. Above a homogeneous ground model, the signal observed at the receiver is solely due to the buried target. However, this is not a realistic situation and the effects of ground inhomogeneities, especially surface disorder; should be studied in order to firmly comment on the overall performance of the TR'T configuration. For this reason, a simulation setup governing a hetcrogeneous ground model, which is displayed in Fig. 4, and a TRTconfigured GPR model is designed. The ground model has a permittivity of $8 \epsilon_{0}$ and a conductivity of $0.01 \mathrm{~S} / \mathrm{n}$. There are 40 holes on the ground-air interface, modeling the surface disorder. Moreover, there are 40 highly conducting small objects in the middle level of the ground and 80 other small scatterers in the lower level of the gronnd. The sizes, locations, permittivities, and conductivities of these ohjects are randonly selected.

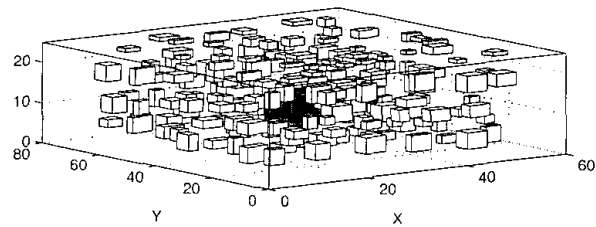

Figure 4: The heterogencous ground model.

First, the simulation resultis of the same dielectric disk, buried in a homogenows ground, is displayed in Fig. 5 in order to provide a comparison with the heterogeneous-ground simulation results. The radar unit travels on a linear path and stops at a point whose projection is on the center of the dielectric disk. The scattered-field image demonstrates that the buried target is easily detected when buried in a honogeneous ground. In Fig. 5, two extra encrgy plots are given, which display the scattered encrgy values with respect to the radar position and tinne-step value.

Figure $6(a)$, which presents the simulation results of the dielectric disk with the heterogeneous ground model, demonstrates that the signals scattered from the clisk are still visible, although a large noise is observed at the early time steps of the B-scan. This noise is absent in the B-scan results obtained with the homogeneous gromutl, in Fig. 5. In order to investigate the characteristics of this noise, the heterogeneous ground model is altered and another simulation is performed. In this new ground model, the surface holes in the previous model are moved one cell into the ground, and therefore, the ground-air interface is regularized. The simulation results of this ground model are displayed in Fig. 6(b). Comparison of Fig. 6(a) and 6(b) reveals that the large eariy-time noise in the results of heterogeneous ground with surface disorder are degraded in the results of the heterogeneous sround with regular ground-air interface. Therefore, it is possible conclude that the TRT-configured GPR is sensitive to surface roughness and the main source of noise is these detoriorations in the ground-air interface. 


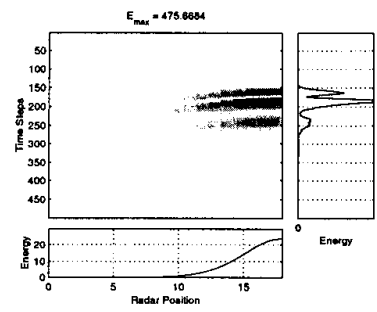

Figure 5: The simulation results of a dielectric disk obtained with a homogeneous ground model.

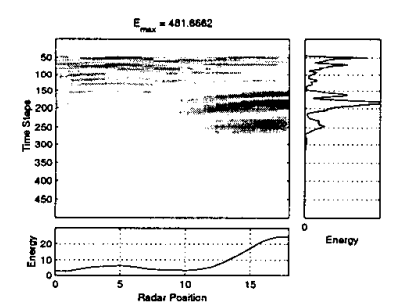

(a)

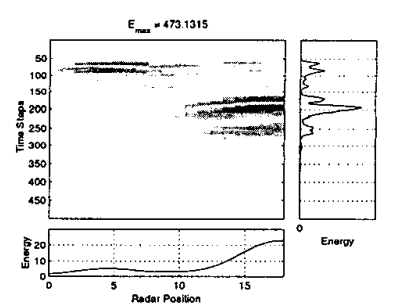

(b)

Figure 6: The simulation results of a dielectric disk obtained with a heterogeneous ground model that contains (a) surface disorder and (b) no surface disorder.

\section{Concluding Remarks}

The TRT configuration of the GPR is a powerful tool to degrade the large undesired signals that dominate the total-received signal. Ground inhomogeneities, especially the ones in the surface of the ground, influence the measurements. However, the signals scattered from the target are still observed at the receiver.

\section{References}

[1] J. M. Bourgeois and G. S. Smith, "A complete electromagnetic simulation of the separated-aperture sensor for detecting buried land mines," IEEE Trans. Antennas Propagat., vol. 46, no. 10, pp. 1419-1426, Oct. 1998.

[2] U. Oğuz and L. Gürel, "Modeling of ground-penetrating-radar antennas with shields and simulated absorbers," IEEE Trans. Antennas Propagat., accepted, Nov. 2000.

[3] P. Luneau and G. Y. Delisle, "Underground target probing using FDTD," 1996 IEEE AP-S International Symposium and URSI Radio Science Meeting, Baltimore, Maryland, U.S.A., pp. 1664-1667, July 1996.

[4] L. Gürel and U. Oğuz, "Three-dimensional FDTD modeling of a ground-penetrating radar," IEEE Trans. Geoscience and Remote Sensing, vol. 38, no. 4, pp. 1513-1521, July 2000 . 